Characterizing the interactions of dietary condensed tannins with bile salts

Xiangxin Li, Wenxiao Jiao, Wanli Zhang, Yan Xu, Jiankang Cao, Weibo Jiang*

College of Food Science and Nutritional Engineering, China Agricultural University, 17 Qinghuadonglu Road, Beijing 100083, P.R. China.

*Corresponding author: Weibo Jiang (Tel.: +86 010-62736565; Fax: +86 01062736565; E-mail: jwb@cau.edu.cn) 
SUPPORTING INFORMATION

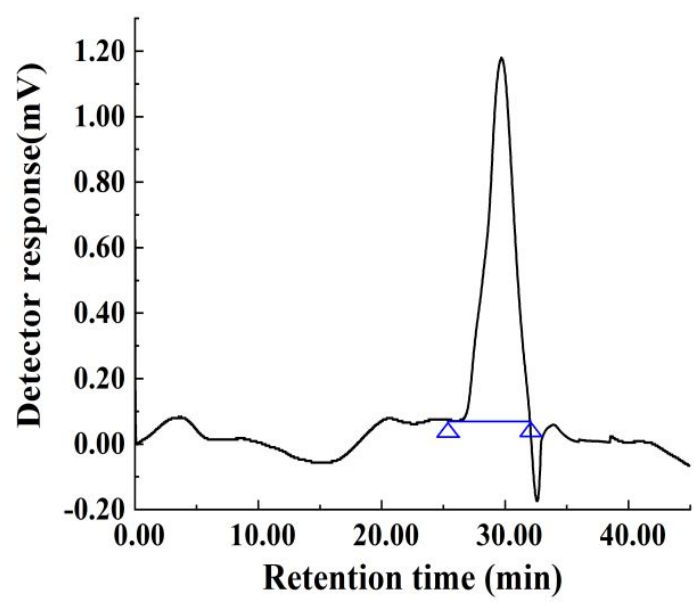

Fig. S1. Gel permeation chromatography analysis of aimed condensed tannins 
A

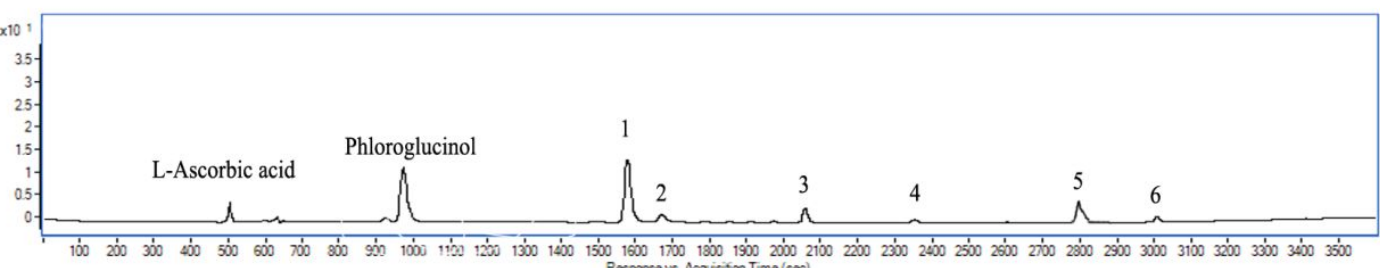

$\mathrm{B}$

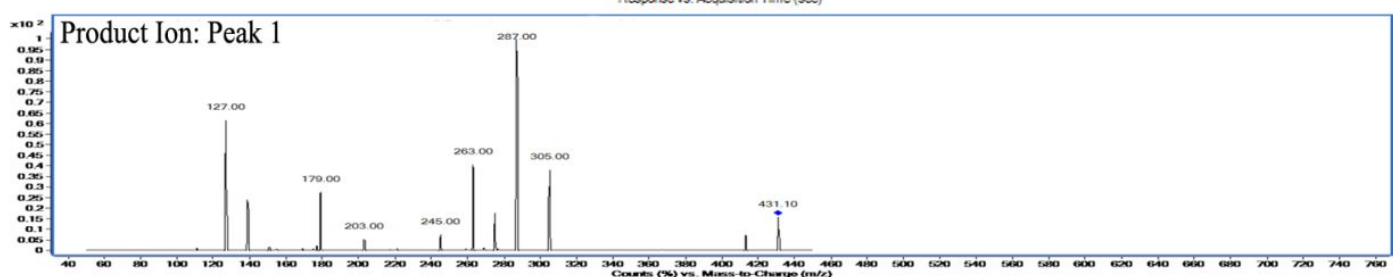

C

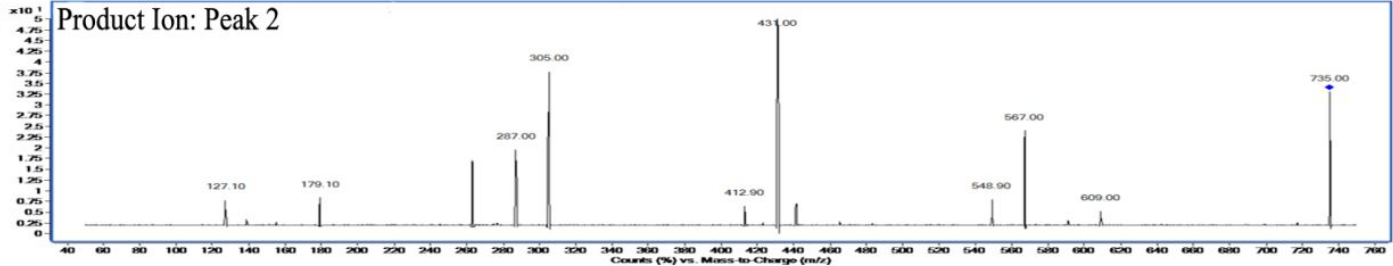

D ㅈa: $>$ Product Ion: Peak 3

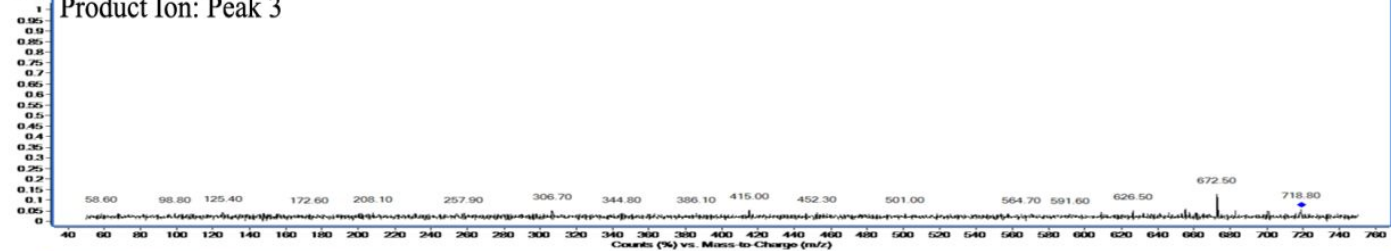

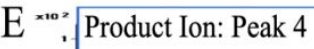

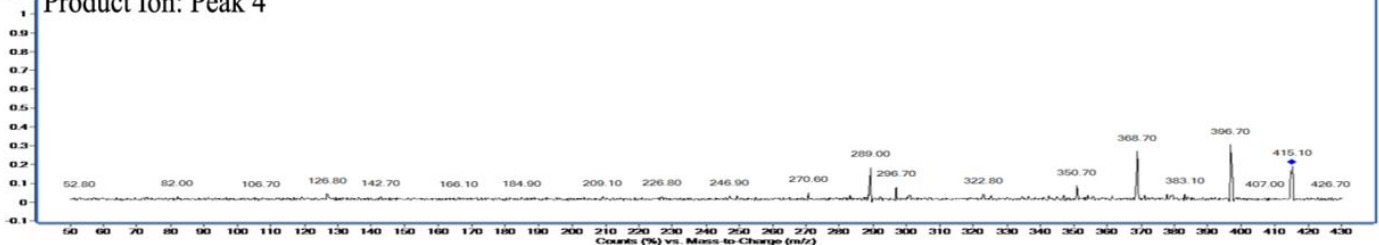

$\mathrm{F} \times 102$, Product Ion: Peak 5

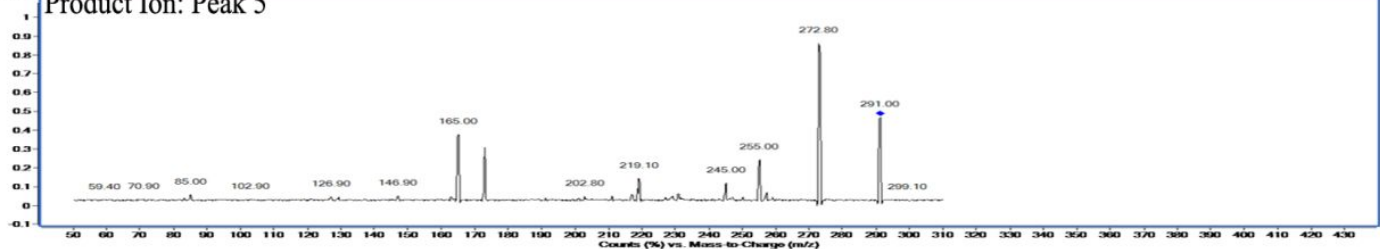

$\mathrm{G} \times$ xo:, Product Ion: Peak 6

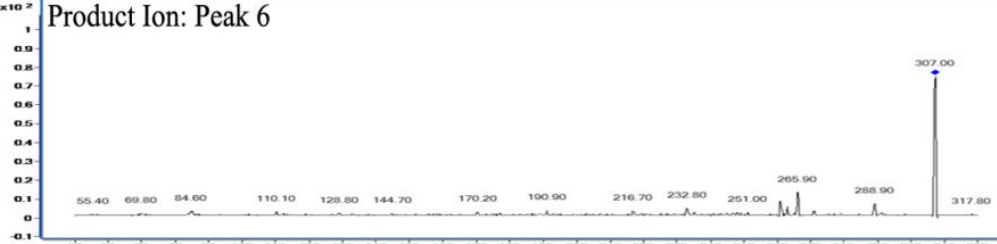

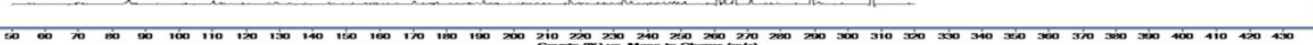

Fig. S2. HPLC profile (A) and MS traces (B, C, D, E, F, G) of the degradation products after depolymerization of aimed condensed tannins with phloroglucinol at 280 nm. Note: Peak 1: EGC-Ph; Peak 2: EGC-EGC-Ph; Peak 3: EGC-EC-Ph; Peak 4: EC-Ph; Peak 5: EC; Peak 6: EGC. 


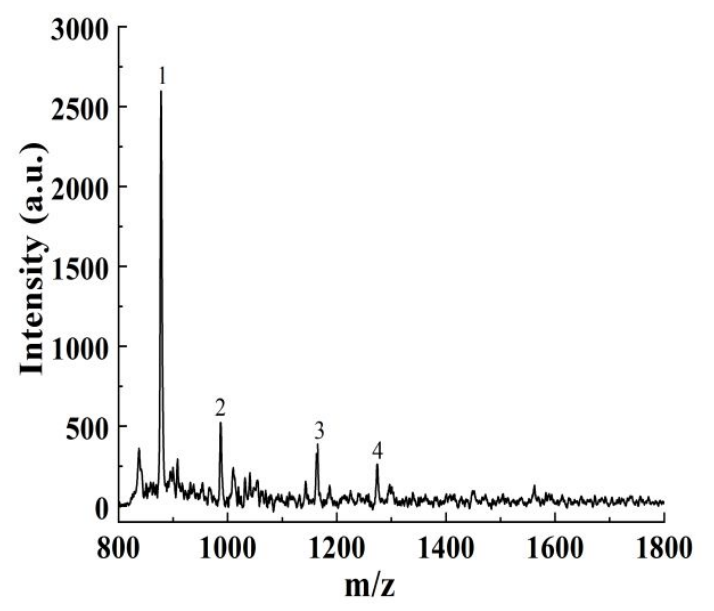

Fig. S3. MALDI-TOF analysis of aimed condensed tannins. Peak 1: 877.06 Da (two EC+EGC); Peak 2: 987.59 Da (three EC +Cs); Peak 3: 1164.34 Da (three EC+EGC); Peak 4: 1274.92 Da (four EC+Cs). 
According to the method of creaming stability and digestion properties, the emulsion was prepared by using $0.05 \%$ Tween- $80(\mathrm{v} / \mathrm{v}$, DDW) as an emulsifier replaced $0.5 \% \mathrm{NaTC}(\mathrm{w} / \mathrm{v}, \mathrm{DDW})$. The effect of $\mathrm{CT}$ on digestion properties of the emulsion stabilized by Tween- 80 had also been studied

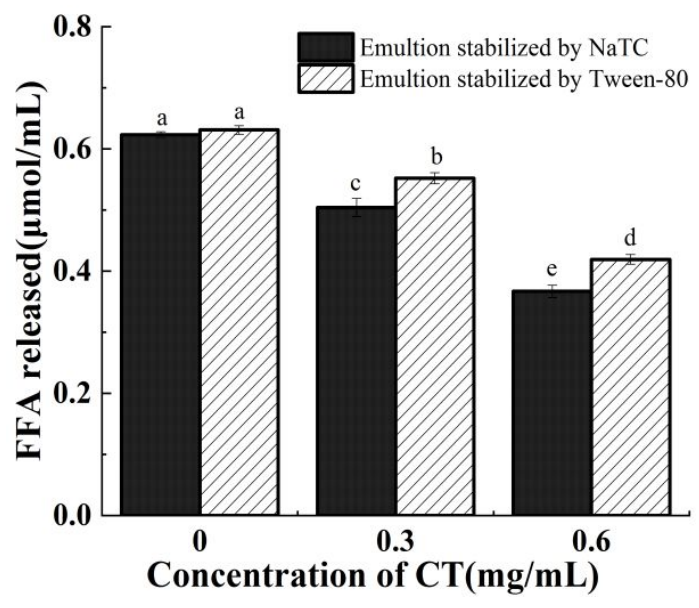

Fig. S4. Effect of CTs on lipid digestion

As shown in Fig.S4, the content of FFA released from the emulsion stabilized by Tween- 80 is higher than that from the emulsion stabilized by NaTC. This indicated that CT not only inhibited the activity of lipase, it also decreased the digestion of emulsion by binding NaTC. 
Table S1. The Critical Micelle Concentration of Bile Salts

\begin{tabular}{cccc}
\hline \multirow{2}{*}{ Bile salt } & \multirow{2}{*}{ MM (Da) } & \multicolumn{2}{c}{ CMC } \\
& & water $^{\mathrm{a}}$ & $0.1 \mathrm{M} \mathrm{PBS}^{\mathrm{b}}$ \\
\hline $\mathrm{NaC}$ & 430.6 & 13 & $9.75 \pm 0.21$ \\
$\mathrm{NaDC}$ & 414.6 & 10 & $6.19 \pm 0.16$ \\
$\mathrm{NaGC}$ & 487.6 & 12 & $9.17 \pm 0.33$ \\
$\mathrm{NaTC}$ & 537.69 & 10 & $8.95 \pm 0.27$ \\
\hline
\end{tabular}

a: cited from reference; ${ }^{b}$ :measured according to the method of conductivity analysis.

\section{Reference cited}

a. Roda, A., Hofmann, A. F., \& Mysels, K. J.. The influence of bile salt structure on self-association in aqueous solutions. Journal of Biological Chemistry 1983, 258(10), 6362-6370.

b. Mahajan, S., \& Mahajan, R. K.. Interactions of phenothiazine drugs with bile salts: micellization and binding studies. Journal of Colloid and Interface Science 2012, 387(1), 194-204. 